Int. J. Morphol.,

33(1):262-266, 2015.

\title{
Polimorfismo del Gen ACTN3 y ECA en Seleccionados de Gimnasia de Brasil y Japón
}

\author{
Gene Polymorphism ACTN3 and ACE in Selected Gymnasts Athletes in Brazil and Japan
}

\begin{abstract}
Andrea Ferreira João*; Alexis Caniuqueo Vargas**; Claudio Hernández Mosqueira***; Sandro Fernandes da Silva ${ }^{* * * *}$; Mikel Izquierdo ${ }^{* * * * *}$; Hector Silva Mella ${ }^{* * * * * *}$; Rodrigo Ramirez-Campillo ${ }^{* * * * * * * *}$ \& José Fernandes Filho ${ }^{* * * * * * * *}$
\end{abstract}

JOÃO, A. F.; CANIUQUEO, V. A; HERNÁNDEZ, M. C.; DA SILVA, S. F.; IZQUIERDO, M.; SILVA, M. H.; RAMIREZCAMPILLO, R. \& FERNANDES FILHO, J. Polimorfismo del gen ACTN3 y ECA en seleccionados de gimnasia de Brasil y Japón. Int. J. Morphol., 33(1):262-266, 2015.

RESUMEN: En el rendimiento deportivo, el estudio de la genética ha cobrado un papel fundamental al momento de estudiar el rendimiento deportivo. El objetivo del estudio fue analizar la frecuencia de genotipo y alelo de a - actinina 3 (ACTN3) R577X y enzima convertidora de angiotensina (ECA) I/D polimorfismo en un grupo de gimnastas de Brasil y Japón. Se considero una muestra de 73 gimnastas (14 de Japón y 59 de Brasil), todos los sujetos firmaron de consentimiento informado. Para la obtención de ACTN3 y ECA se recogió una muestra la saliva y analizaron los genotipos mediante el empleo de cadena de la polimerasa en tiempo real a partir del iQ5 Thermal Cycler, BioRad. Los resultados muestran una prevalencia del genotipo RX ACTN3, prevalencia del alelo R, prevalencia del genotipo DI ECA y prevalencia de alelo D en el total del grupo. Se concluye que el predominio RX del ACTN3, prevalencia del alelo R y predominio del genotipo DI en ECA pueden ofrecer una ventaja genética en cuanto a niveles de fuerza y potencia muscular, posiblemente facilitando la práctica y el éxito competitivo en gimnastas.

PALABRAS CLAVE: Polimorfismo; a-actinina 3; Enzima convertidora de angiotensina; Rendimiento deportivo.

\section{INTRODUCCIÓN}

La formulación de la metodología científica aplicada al proceso de selección deportiva y obtención del máximo rendimiento requiere de la identificación del perfil del atleta de alto rendimiento a través de una evaluación funcional, estructural y genética (García-Pallarés et al., 2011). En el campo de la genética, las investigaciones se han orientado en la búsquedas de perfiles genéticos asociados al rendimiento deportivo de atletas de elite que permitan la orientación del entrenamiento y selección deportiva (Alfred $e t$ al., 2011). Dos genes han sido ampliamente estudiados y asociados al rendimiento deportivo, la enzima convertidora de angiotensina inserción/deleción (ECA I/D) polimorfismo y el gen de la a-actina-3 (ACTN3) polimorfismo R577X (Ahmetov et al., 2013; Alfred et al.; Chiu et al., 2012; Doring et al., 2010).
La ECA, localizado en el cromosoma 17, implicada en el sistema renina angiotensina aldosterona actúa en la regulación del volumen sanguíneo, presión arterial y balance de electrolitos (McCauley et al., 2010). La inserción (I) y deleción (D) como polimorfismo, se ha asociado con mejoras en el rendimiento deportivo otorgando al alelo I de la ECA una asociación con el rendimiento en pruebas de resistencia y al alelo $\mathrm{D}$ un mejor desempeño en pruebas de fuerza y velocidad (McCauley et al.; Rodriguez-Romo et al., 2010).

El gen ACTN3 por su parte, codifica la proteína ACTN3, que pertenece al citoesqueleto muscular y predomina en la fila Z sarcomérica (Niemi \& Majamaa, 2005). El ACTN3 situado en las fibras de contracción rápida

\footnotetext{
Universidad Federal de Río de Janeiro, Río de Janeiro, Brasil.

** Laboratorio de Fisiología y Biomecánica, Universidad Autónoma de Chile, Temuco, Chile.

*** Pedagogía en Educación Física, Universidad Pedro de Valdivia, Chillán, Chile.

***** Universidad Federal de Lavras, Lavras, Brasil.

****** Departamento Ciencias de la Salud, Universidad Pública de Navarra, Navarra, España.

******** Universidad de La Frontera, Temuco, Chile.

******** Department of Physical Activity Sciences, Universidad de Los Lagos, Osorno, Chile.

*********Laboratorio de Biociencias en el Movimiento Humano - EEFD - Universidad Federal de Río de Janeiro, Río de Janeiro, Brasil.
} 
glucolíticas permite generar una alta capacidad contráctil, especialmente importante en los deportes que implican la generación de gran velocidad y potencia muscular. En este sentido, una variación genética de ACTN3 como resultado de la sustitución de una arginina $(\mathrm{R})$ con un codón de parada (X) en el aminoácido 577, puede crear dos versiones diferentes del gen ACTN3 (MacArthur et al., 2008; Seto et al., 2013).

Diversos estudios han demostrado respecto al polimorfismo de ECA, un predominio del alelo D en deportistas que practican deportes de fuerza y velocidad (Pereira et al., 2013; Bustamante-Ara et al., 2010; Delmonico et al., 2007). Por otro lado, respecto al polimorfismo del ACTN3, diversas investigaciones han reportado el predominio del genotipo RR o RX en deportistas de elite en performance de fuerza y velocidad (Bell et al.; Chiu et al., 2011; Erskine et al., 2014). En el campo de la genética en la gimnasia, Morucci et al. (2014) han observado bajas significativas del polimorfismo ACE II respecto a las frecuencia ID y DD, argumentado una disposición del gimnasta hacia condiciones de potencia, sin hallazgos similares en los genotipos RR y RX del ACTN3.

La presente investigación plantea como objetivo analizar la frecuencia de genotipo y alelo ACTN3 R577X y ECA I/D polimorfismo en gimnasta de Brasil y Japón, con el fin de colaborar con antecedentes que permitan construir un perfil genético en esta especialidad deportiva.

\section{MATERIAL Y MÉTODO}

Fueron seleccionados 73 Gimnastas, pertenecientes a selección Juvenil-Adulta de gimnasia artística (G.A.) de Japón (14 Varones), selección G.A. sub-14 de Brasil (12 damas), selección G.A. Juvenil-Adulta adulta de Brasil (10 damas y 23 varones), selección juvenil adulta de gimnasia rítmica (G.R.) de Brasil (14 damas). Todos los participantes firmaron un consentimiento informado y se procedió de acuerdo a la declaración de Helsinki.
El material biológico utilizado para analizar el ACTN3 Polimorfismo R577XY y ECA provenía de las células de la mucosa oral que se recogieron utilizando un bastoncillo de algodón estéril a través de la saliva. Para su análisis, la muestra fue enviada al laboratorio forense de ADN del Instituto de Policía Científica de Paraíba-SPI/PB/BR, Brasil y Helixxa Servicios Genómicos - División de Deporte - Laboratorio de Apoyo - Campinas - SP, Brasil.

Las muestras recogidas se sometieron a extracción de ADN de acuerdo con la procedimientos descritos por Walsh et al. (1991). Los genotipos fueron determinado mediante el empleo de cadena de la polimerasa en tiempo real con el equipo IQ5 Thermal Cycler (Bio-Rad Laboratories, Inc.) con un kit para determinar el polimorfismo R577X y polimorfismo ECA D/I, de acuerdo a las instrucciones del fabricante. Para el análisis de los datos, se utilizó estadística descriptiva considerando las medidas de frecuencia de aparición de genotipo y alelos, mediante el programa SPSS versión 18.0 .

\section{RESULTADOS}

El genotipo RX ACTN3 fue el que obtuvo una mayor frecuencia de aparición en el total de los gimnastas evaluados. Comportamiento similar se observa en cada uno de las diferentes categorías grupales, excepto el grupo de gimnasia artística menores de 14 años de Brasil cuya mayor frecuencia genotípica se observa en el genotipo XX. En relación a la frecuencia de aparición de alelos, el X presenta una mayor frecuencia sólo en las damas sub 14 de gimnasia artística (Tabla I).

En relación a ECA, el genotipo DI obtuvo una mayor frecuencia en los sujetos evaluados. Sin embargo, las especialistas de gimnasia rítmica de Brasil presentaron un mayor predominio del genotipo DD.

En relación a la frecuencia de aparición de alelos, el mayor predominio del alelo D se registró en los gimnastas

Tabla I. Frecuencias de genotipo y alelo de ACTN3 R577X polimorfismo.

\begin{tabular}{|c|c|c|c|c|c|c|c|c|c|c|}
\hline \multirow{2}{*}{ Sujetos (n) } & \multicolumn{6}{|c|}{ Frecuencia de genotipo (n) } & \multicolumn{4}{|c|}{ Frecuencia de Alelo (n) } \\
\hline & \multicolumn{2}{|c|}{$\mathbf{R R}$} & \multicolumn{2}{|c|}{$\mathbf{R X}$} & \multicolumn{2}{|c|}{$\mathbf{X X}$} & \multicolumn{2}{|c|}{$\mathbf{R}$} & \multicolumn{2}{|c|}{$\mathbf{X}$} \\
\hline G. A. Damas < 14 años Brasil (12) & 4 & $33 \%$ & 2 & $17 \%$ & 6 & $50 \%$ & 10 & $42 \%$ & 14 & $58 \%$ \\
\hline G. A. Damas Juvenil-adultos Brasil (10) & 4 & $40 \%$ & 6 & $60 \%$ & 0 & $0 \%$ & 14 & $70 \%$ & 6 & $30 \%$ \\
\hline G. A. Varones Juvenil-adultos. Brasil (23) & 5 & $22 \%$ & 13 & $57 \%$ & 5 & $22 \%$ & 23 & $50 \%$ & 23 & $50 \%$ \\
\hline G. R. Brasil (14) & 3 & $21 \%$ & 8 & $57 \%$ & 3 & $21 \%$ & 14 & $50 \%$ & 14 & $50 \%$ \\
\hline G. A. Varones Japón Juvenil - Adultos (14) & 3 & $21 \%$ & 9 & $64 \%$ & 2 & $14 \%$ & 15 & $54 \%$ & 13 & $46 \%$ \\
\hline Total (73) & 19 & $26 \%$ & 38 & $52 \%$ & 16 & $22 \%$ & 76 & $52 \%$ & 70 & $48 \%$ \\
\hline
\end{tabular}


brasileños, a excepción de las damas sub 14 de la gimnasia artística en quienes predominio del alelo I. Esta última ca- racterística, también se observó en los gimnastas de Japón (Tabla II).

Tabla II. Frecuencias de genotipo y alelo de ECA I/D polimorfismo.

\begin{tabular}{|c|c|c|c|c|c|c|c|c|c|c|}
\hline \multirow{2}{*}{ Sujetos (n) } & \multicolumn{6}{|c|}{ Frecuencia de genotipo, n y \% } & \multicolumn{4}{|c|}{ Frecuencia de Allelo, n y \% } \\
\hline & \multicolumn{2}{|c|}{ DD } & \multicolumn{2}{|c|}{ DI } & \multicolumn{2}{|c|}{ II } & \multicolumn{2}{|c|}{ D } & \multicolumn{2}{|c|}{ I } \\
\hline G. A. Damas < 14 años Brasil (12) & 1 & $8 \%$ & 8 & $67 \%$ & 3 & $25 \%$ & 10 & $42 \%$ & 14 & $58 \%$ \\
\hline G. A. Damas Juvenil-adultos (10) & 3 & $30 \%$ & 6 & $60 \%$ & 1 & $10 \%$ & 12 & $60 \%$ & 8 & $40 \%$ \\
\hline G. A. Varones Juvenil-adultos. Brasil (23) & 8 & $35 \%$ & 11 & $48 \%$ & 4 & $17 \%$ & 27 & $59 \%$ & 19 & $41 \%$ \\
\hline G. R. Brasil (14) & 8 & $57 \%$ & 6 & $43 \%$ & 0 & $0 \%$ & 22 & $79 \%$ & 6 & $21 \%$ \\
\hline G. A. Varones Japón Juvenil - Adultos (14) & 2 & $14 \%$ & 6 & $43 \%$ & 6 & $43 \%$ & 10 & $36 \%$ & 18 & $64 \%$ \\
\hline Total (73) & 22 & $30 \%$ & 37 & $51 \%$ & 14 & $19 \%$ & 81 & $55 \%$ & 65 & $45 \%$ \\
\hline
\end{tabular}

\section{DISCUSIÓN}

Uno de los principales resultados del trabajo fue el predominio del genotipo $\mathrm{RX}$ respecto al ACTN3 en gimnastas de Brasil y Japón. Este predominio se ha observado también en atletas luchadores de elite de alto rendimiento y también de rendimiento a nivel nacional (Kikuchi et al., 2012). Diversos estudios señalan un predominio del genotipo RX en atletas de alto rendimiento de deportes caracterizados por condiciones de fuerza y velocidad, Ruiz et al. (2011) observó una frecuencia de genotipo RR de 31,1\% y RX de 50,6\%, Norman et al. (2009) RR de 31\% y RX de $50 \%$, Niemi \& Majamaa RR de 45,0\% y RX de 45,8\%, Yang et al. (2003) RR de 30,0\% y RX de 52,0\% y Druzhevskaya et al. (2008) RR de 36,8\% y RX de 49,0\%. Sin embargo, algunos investigadores observaron una mayor proporción de genotipo RR como los estudios de McCauley et al. RR de 43,0\% y RX de 41,0\%, y Scott et al. (2010) RR de 75,0\% y RX de 23,0\% en Jamaica y RR de 66,0\% y RX de 30,0\% en afroamericanos de Estados Unidos.

En relación a la frecuencia de aparición del alelo en ACTN3, se ha reportado una mayor frecuencia del alelo $\mathrm{R}$ en deportes de potencia y velocidad (Kikuchi et al.) similar a lo registrado por en los gimnastas en este estudio.

Las diferencia de frecuencia de aparición genotipo DI/ DD podría estar vinculada a las características específicas de su especialidad, ya que el predominio del alelo D se ha asociado a una mayor actividad de ECA, y ha sido reportado como el alelo de mayor predominio en deportes de potencia, como luchadores de elite (Kikuchi et al.), atletas de velocidad (Niemi \& Majamaa; MacArthur \& North; Scott et al.) y nadadores de elite en distancias cortas y atletas de velocidad (Costa et al., 2009). El predomino del alelo I reportado en gimnastas japonés, también ha sido registrado en estudios realizados en deportistas de resistencia (Costa et al.; McCauley et al.), pudiendo sufrir de variaciones en función del sexo.

\section{CONCLUSIÓN}

Las características multifactoriales del rendimiento deportivo, permiten establecer características genéticas de una especialidad deportiva de acuerdo a los resultados reportado por diversas investigaciones en ECA y ACTN3.

De acuerdo a lo anterior, los resultados encontrados en el presente estudio, permiten establecer el predominio del genotipo RX del ACTN3 y una mayor frecuencia de aparición del alelo $\mathrm{R}$, características que se han perfilado en la literatura para deportes de fuerza y velocidad.

Respecto al comportamiento de ECA en los gimnasta en la muestra evaluada, el genotipo DI se sitúa como predominante, evidenciando una mayor frecuencia de aparición del alelo D en el grupo de gimnasia artística brasileña y cuyo predominio se asocia a características de potencia. El alelo I, asociado a deportes de resistencia fue de mayor predomino en el grupo de gimnasia rítmica brasileña y gimnastas japoneses.

Si bien se requieren más investigaciones que permitan aclarar el papel de ACTN3 y ECA en el rendimiento de gimnastas, los resultados pueden sugeridos para identificar el potencial genético en deportistas de la especialidad.

JOÃO, A. F.; CANIUQUEO, V. A; HERNÁNDEZ, M. C.; DA SILVA, S. F.; IZQUIERDO, M.; SILVA, M. H.; RAMIREZCAMPILLO, R. \& FERNANDES FILHO, J. Gene polymorphism ACTN3 and ACE in selected gymnasts in Brazil and Japan. Int. J. Morphol., 33(1):262-266, 2015.

SUMMARY: In the field of competitive sport, genetics has gained a fundamental role in the study of sport performance. The aim of this study was to analyze the prevalence of polymorphisms R577X and insertion/deletion (I/D), occurring in a-actinin-3 (ACTN3) and angiotensin converting enzyme (ACE) genes, respectively, in Brazilian and Japanese gymnasts. A suitable non-probabilistic sample 
of 73 gymnasts (14 from Japan) was recruited and signed an informed consent. To measure ACTN3 and ECA saliva samples were obtained by means of real time polymerase chain reaction (iQ5 Thermal Cycler, BioRad). A high prevalence of RX ACTN3 genotype, R allele, ACE I/D genotype, and D allele were observed in Brazilian and Japanese gymnasts. In conclusion a high prevalence of RXACTN3 genotype, $\mathrm{R}$ allele and ACE I/D genotype would allow a genetic advantage regarding muscle strength and power, possibly facilitating competitive success in gymnastics.

KEY WORDS: Polymorphism; Angiotensin converting enzyme; a-actinin-3; Sport performance.

\section{REFERENCIAS}

Ahmetov, I. I.; Gavrilov, D. N.; Astratenkova, I. V.; Druzhevskaya, A. M.; Malinin, A. V.; Romanova, E. E. \& Rogozkin, V. A. The association of ACE, ACTN3 and PPARA gene variants with strength phenotypes in middle school-age children. $J$. Physiol. Sci., 63(1):79-85, 2013.

Alfred, T.; Ben-Shlomo, Y.; Cooper, R.; Hardy, R.; Cooper, C.; Deary, I. J; Gunnell, D.; Harris, S. E.; Kumari, M.; Martin, R. M.; Moran, C. N.; Pitsiladis, Y. P.; Ring, S. M.; Sayer, A. A.; Smith, G. D.; Starr, J. M.; Kuh, D.; Day, I. N. \& HALCyon study team. ACTN3 genotype, athletic status, and life course physical capability: meta-analysis of the published literature and findings from nine studies. Hum. Mutat., 32(9):1008-18, 2011.

Bell, W.; Colley, J. P.; Evans, W. D.; Darlington, S. E. \& Cooper, S. M. ACTN3 genotypes of Rugby Union players: distribution, power output and body composition. Ann. Hum. Biol., 39(1):19-27, 2012.

Bustamante-Ara, N.; Santiago, C.; Verde, Z.; Yvert, T.; GómezGallego, F.; Rodríguez-Romo, G.; González-Gil, P.; SerraRexach, J. A.; Ruiz, J. R. \& Lucia, A. ACE and ACTN3 genes and muscle phenotypes in nonagenarians. Int. J. Sports Med., 31(4):221-4, 2010.

Costa, A. M.; Silva, A. J.; Garrido, N. D.; Louro, H.; de Oliveira, R. J. \& Breitenfeld, L. Association between ACE D allele and elite short distance swimming. Eur. J. Appl. Physiol., 106(6):785-90, 2009.

Chiu, L. L.; Chen, T. W.; Hsieh, S. S. \& Hsieh, L. L. ACE I/D, ACTN3 R577X, PPARD T294C and PPARGC1A Gly482Ser polymorphisms and physical fitness in Taiwanese late adolescent girls. J. Physiol. Sci., 62(2):115-21, 2012.

Chiu, L. L.; Wu, Y. F.; Tang, M. T.; Yu, H. C.; Hsieh, L. L. \& Hsieh, S. S. ACTN3 genotype and swimming performance in Taiwan. Int. J. Sports Med., 32(6):476-80, 2011.
Delmonico, M. J.; Kostek, M. C.; Doldo, N. A.; Hand, B. D.; Walsh, S.; Conway, J. M.; Carignan, C. R.; Roth, S. M. \& Hurley, B. F. Alpha-actinin-3 (ACTN3) R577X polymorphism influences knee extensor peak power response to strength training in older men and women. J. Gerontol. A Biol. Sci. Med. Sci., 62(2):20612, 2007.

Druzhevskaya, A. M.; Ahmetov, I. I.; Astratenkova, I. V. \& Rogozkin, V. A. Association of the ACTN3 R577X polymorphism with power athlete status in Russians. Eur. $J$. Appl. Physiol., 103(6):631-4, 2008.

Erskine, R. M.; Williams, A. G.; Jones, D. A.; Stewart, C. E. \& Degens, H. The individual and combined influence of ACE and ACTN3 genotypes on muscle phenotypes before and after strength training. Scand. J. Med. Sci. Sports, 24(4):642-8, 2014.

García-Pallarés, J.; López-Gullón, J. M.; Muriel, X.; Díaz, A. \& Izquierdo, M. Physical fitness factors to predict male Olympic wrestling performance. Eur. J. Appl. Physiol., 111(8):1747-58, 2011.

Kikuchi, N.; Min, S. K.; Ueda, D.; Igawa, S. \& Nakazato, K. Higher frequency of the ACTN3 R allele + ACE DD genotype in Japanese elite wrestlers. J. Strength Cond. Res., 26(12):327580, 2012.

MacArthur, D. G.; Seto, J. T.; Chan, S.; Quinlan, K. G.; Raftery, J. M.; Turner, N.; Nicholson, M. D.; Kee, A. J.; Hardeman, E. C.; Gunning, P. W.; Cooney, G. J.; Head, S. I.; Yang, N. \& North, K. N. An Actn3 knockout mouse provides mechanistic insights into the association between alpha-actinin-3 deficiency and human athletic performance. Hum. Mol. Genet., 17(8):1076-86, 2008.

McCauley, T.; Mastana, S. S. \& Folland, J. P. ACE I/D and ACTN3 $\mathrm{R} / \mathrm{X}$ polymorphisms and muscle function and muscularity of older Caucasian men. Eur. J. Appl. Physiol., 109(2):269-77, 2010 .

Morucci, G.; Punzi, T.; Innocenti, G.; Gulisano, M.; Ceroti, M. \& Pacini, S. New frontiers in sport training: genetics and artistic gymnastics. J. Strength Cond. Res., 28(2):459-66, 2014.

Niemi, A. K. \& Majamaa, K. Mitochondrial DNA and ACTN3 genotypes in Finnish elite endurance and sprint athletes. Eur. J. Hum. Genet., 13(8):965-9, 2005.

Norman, B.; Esbjörnsson, M.; Rundqvist, H.; Osterlund, T.; von Walden, F. \& Tesch, P. A. Strength, power, fiber types, and mRNA expression in trained men and women with different ACTN3 R577X genotypes. J. Appl. Physiol. (1985), 106(3):959-65, 2009.

Pereira, A.; Costa, A. M.; Leitão, J. C.; Monteiro, A. M.; Izquierdo, M.; Silva, A. J.; Bastos, E. \& Marques, M. C. The influence of ACE ID and ACTN3 R577X polymorphisms on lowerextremity function in older women in response to highspeed power training. BMC Geriatr., 13:131, 2013. 
Rodriguez-Romo, G.; Ruiz, J. R.; Santiago, C.; Fiuza-Luces, C.; González-Freire, M.; Gómez-Gallego, F.; Morán, M. \& Lucia, A. Does the ACE I/D polymorphism, alone or in combination with the ACTN3 R577X polymorphism, influence muscle power phenotypes in young, non-athletic adults? Eur. J. Appl. Physiol., 110(6):1099-106, 2010.

Ruiz, J. R.; Fernández del Valle, M.; Verde, Z.; Díez-Vega, I.; Santiago, C.; Yvert, T.; Rodríguez-Romo, G.; Gómez-Gallego, F.; Molina, J. J. \& Lucia, A. ACTN3 R577X polymorphism does not influence explosive leg muscle power in elite volleyball players. Scand. J. Med. Sci. Sports, 21(6):e34-41, 2011.

Scott, R. A.; Irving, R.; Irwin, L.; Morrison, E.; Charlton, V.; Austin, K.; Tladi, D.; Deason, M.; Headley, S. A.; Kolkhorst, F. W.; Yang, N.; North, K. \& Pitsiladis, Y. P. ACTN3 and ACE genotypes in elite Jamaican and US sprinters. Med. Sci. Sports Exerc., 42(1):107-12, 2010.

Seto, J. T.; Quinlan, K. G.; Lek, M.; Zheng, X. F.; Garton, F.; Macarthur, D. G.; Hogarth, M. W.; Houweling, P. J.; Gregorevic, P.; Turner, N.; Cooney, G. J.; Yang, N. \& North, K. N. ACTN3 genotype influences muscle performance through the regulation of calcineurin signaling. J. Clin. Invest., 123(10):4255-63, 2013.

Walsh, P. S.; Metzger, D. A. \& Higuchi, R. Chelex 100 as a medium for simple extraction of DNA for PCR-based typing from forensic material. Biotechniques, 10(4):506-13, 1991.

Yang, N.; MacArthur, D. G.; Gulbin, J. P.; Hahn, A. G.; Beggs, A. H.; Easteal, S. \& North, K. ACTN3 genotype is associated with human elite athletic performance. Am. J. Hum. Genet., 73(3):627-31, 2003

\author{
Dirección para Correspondencia: \\ Alexis Caniuqueo Vargas, PhD \\ Académico \\ Universidad Autónoma de Chile \\ Avenida Alemania 01090 \\ Temuco \\ CHILE
}

Email: alexis.caniuqueo@uautonoma.cl

Recibido: 17-10-2014

Aceptado: 30-12-2014 